\title{
Atividades físicas de lazer e transtornos mentais comuns em jovens de Feira de Santana, Bahia
}

\author{
Leisure physical activities and common mental disorders among young people in Feira \\ de Santana, Bahia, Brazil
}

\author{
Luzana Cirqueira Rios ${ }^{1}$, Maura Maria Guimarães de Almeida ${ }^{2}$, Saulo Vasconcelos Rocha ${ }^{3}$, Tânia Maria \\ Araújo $^{4}$, Paloma de Sousa Pinho
}

${ }^{1}$ Enfermeira. Ex-bolsista, Iniciação Científica, Núcleo de Epidemiologia (NEPI), Universidade Estadual de Feira de Santana (UEFS), Feira de Santana, BA. ${ }^{2}$ Doutora em Enfermagem. Professora titular, Departamento de Saúde, UEFS. Pesquisadora, NEPI, UEFS. ${ }^{3}$ Mestre em Saúde Coletiva. Professor assistente, Departamento de Saúde, Universidade Estadual do Sudoeste da Bahia (UESB), Vitória da Conquista, BA. Bolsista da Coordenação de Aperfeiçoamento de Pessoal de Nível Superior (CAPES). ${ }^{4}$ Doutora em Epidemiologia. Professora titular, Departamento de Saúde, UEFS. Pesquisadora, NEPI, UEFS. ${ }^{5}$ Mestre em Saúde Coletiva. Professora assistente, Centro de Ciências da Saúde, Universidade Federal do Recôncavo Baiano (UFRB), Cruz das Almas, BA. Pesquisadora, NEPI, UEFS.

\section{Resumo}

Introdução: Os transtornos mentais comuns (TMC) são transtornos comumente encontrados na população e resultam do estilo de vida moderno. As atividades físicas de lazer podem diminuir as tensões, evitando esses transtornos.

Objetivo: Estimar a prevalência de TMCs em jovens que participam ou não de atividades físicas de lazer em Feira de Santana (BA).

Métodos: Estudo epidemiológico, de corte transversal, incluindo 3.597 indivíduos, entre os quais 1.400 jovens com idade entre 15 e 29 anos, residentes na zona urbana de Feira de Santana. As atividades físicas de lazer foram avaliadas por meio de um questionário estruturado que abordou frequência, tipo, motivo e esforço envolvido em cada atividade. O Self-Reporting Questionnaire (SRQ-20) foi utilizado para avaliar os TMCs.

Resultados: A prevalência de TMC foi de 19,2\% entre os adolescentes-jovens (15-17 anos), 26,6\% entre os jovens-jovens (18-24 anos) e 27,8\% entre os jovens-adultos (25-29 anos). Quanto à avaliação das atividades de lazer, 89,9\% disseram que mantinham algum tipo de atividade, porém percebeu-se uma diminuição da prática de atividades físicas de lazer com o avançar da idade. Ao analisar a relação entre atividades físicas de lazer e TMC, foi verificada associação negativa (razão de prevalência: 0,59, intervalo de confiança de 95\%: 0,51-0,67).

Conclusão: Nos programas de atenção a saúde devem ser incluídas ações que favoreçam a prática de atividades físicas de lazer, tendo em vista a contribuição do comportamento ativo na melhoria da saúde mental.

Descritores: Transtornos mentais, atividade física de lazer, saúde mental.

\begin{abstract}
Introduction: Common mental disorders (CMD) are disorders commonly found in the population. They are a consequence of modern lifestyle. Leisure physical activities can reduce tension and therefore help prevent CMDs.

Objective: To estimate the prevalence of CMDs among youths who engage and those who do not engage in leisure physical activities in Feira de Santana (BA), Brazil.

Methods: A cross-sectional, epidemiological study was conducted with 3,597 individuals, including 1,400 young people aged 15-29 years, living in the urban area of Feira de Santana. Leisure physical activities were assessed using a structured questionnaire covering the frequency, type, reason and effort involved in each activity. The Self-Reporting Questionnaire (SRQ-20) was used to assess CMDs.

Results: The prevalence of CMD was 19.2\% among adolescents (15-17 years old), 26.6\% among youths (18-24 years), and $27.8 \%$ among young adults (25-29 years). In the assessment of leisure activities, $89.9 \%$ of the youths reported to regularly engage in some kind of activity; however, engagement in leisure physical activities was observed to reduce with age. A negative association was found between leisure physical activities and CMD (prevalence ratio: $0.59,95 \%$ confidence interval: 0.51-0.67).

Conclusion: Healthcare programs should include actions that encourage leisure physical activities, in view of the contribution of active behavior toward improving mental health.

Keywords: Mental disorders, leisure physical activities, mental health.
\end{abstract}

\section{Correspondência:}

Luzana Cirqueira Rios, Núcleo de Epidemiologia, Departamento de Saúde, Universidade Estadual de Feira de Santana, BR-116, km 3, Campus Universitário, Módulo VI, Centro de Pós-Graduação em Saúde Coletiva, CEP 44031-460, Feira de Santana, BA, Brasil. E-mail: luzanacr@hotmail.com

Não foram declarados conflitos de interesse associados à publicação deste artigo.

Copyright (C) Revista de Psiquiatria do Rio Grande do Sul - APRS

Recebido 08/10/2009. Aceito em 04/02/2010.

Rev Psiquiatr Rio Gd Sul. 2011;33(2): 98-102 


\section{Introdução}

As situações e os estilos de vida da sociedade moderna acabam gerando, com muita frequência, situações de ansiedade e alterações mentais (transitórias ou permanentes) suficientes para retirar de algumas pessoas a serenidade mental e/ou a capacidade de decisão necessária aos atos da vida cotidiana.

Em 1992, Goldberg \& Huxley ${ }^{1}$ criaram a expressão transtornos mentais comuns (TMC) para designar morbidade psíquica comumente encontrada na população e que poderia estar relacionada ao estilo de vida moderno. Entre os TMC, é possível citar o estresse, a ansiedade, a insônia, a fadiga, a irritabilidade e o esquecimento, além de queixas somáticas, como cefaleia e má digestão - fatores esses que impossibilitam ao indivíduo manter o bom funcionamento da mente e, consequentemente, do seu organismo em geral.

As atividades físicas de lazer atuam como mecanismos compensatórios frente ao estresse, à angústia, à ansiedade e a outros transtornos mentais, agindo como aliviadores das tensões, renovando as energias e proporcionando prazer, relaxamento e bem-estar aos seus praticantes.

A prática de atividades físicas de lazer e os benefícios gerados por essa prática na saúde dos adolescentes e adultos jovens são assuntos ainda pouco explorados no meio científico. Estudos já publicados apresentam limitações no que se refere à investigação sobre as dimensões significativas da vivência dos jovens e sua relação com a cultura, a religião, o lazer e o esporte 2 .

Assim, o presente estudo teve como objetivo estimar a prevalência de TMC em jovens que participam ou não de atividades físicas de lazer em Feira de Santana (BA). Espera-se que os resultados desta pesquisa possam contribuir para um melhor entendimento da relação entre atividade física de lazer e saúde mental na população jovem do município, oferecendo, assim, subsídios para a elaboração de políticas públicas e estratégias com vistas à promoção da saúde desses jovens.

\section{Material e métodos}

Realizou-se um estudo epidemiológico, de corte transversal, com amostra representativa da população urbana de Feira de Santana (BA), com idade igual ou superior a 15 anos. As áreas selecionadas foram determinadas por amostragem estratificada por subdistrito mediante critérios aleatórios, a partir de dados censitários do Instituto Brasileiro de Geografia e Estatística (IBGE).

O município de Feira de Santana está localizado na região da zona de planície, entre o Recôncavo e os tabuleiros semiáridos do nordeste baiano, e tem uma população de 535.820 habitantes $^{3}$. O município encontra-se dividido em subdistritos, os quais integram setores censitários: cada setor censitário possui ruas, e cada rua possui domicílios. As áreas de estudo incluíram os cinco subdistritos da zona urbana de Feira de Santana (Mangabeira: 40 setores; Pampalona: 42 setores; Santana: 144 setores; Santo Antônio: 68 setores; Su- baé: 47 setores) $)^{3}$. Em cada subdistrito, foram selecionados, por procedimento aleatório, os setores censitários a serem incluídos no estudo; em cada setor, foram selecionadas as ruas; nas ruas selecionadas, todos os domicílios foram incluídos na amostra. Todas as pessoas com 15 anos de idade ou mais residentes nos domicílios sorteados foram consideradas elegíveis para o estudo.

Os dados analisados incluíram um total de 3.597 indivíduos com idade igual ou superior a 15 anos. No entanto, para o presente estudo, a população-alvo selecionada foi de 1.400 indivíduos jovens com idade entre 15 e 29 anos, de acordo com classificação etária do Conselho Nacional de Juventude ${ }^{2}$ para adolescentes-jovens (entre 15 e 17 anos), jovens-jovens (entre 18 e 24 anos) e jovens-adultos (entre 25 e 29 anos).

Foram conduzidas entrevistas domiciliares para a triagem de jovens suspeitos de portar algum tipo de transtorno mental, a partir das áreas com alta prevalência de transtornos mentais em Feira de Santana. Para a triagem, foram utilizados dois instrumentos de pesquisa: uma ficha domiciliar e um questionário individual que incluía informações sociodemográficas, características do trabalho profissional e doméstico, aspectos da saúde reprodutiva, informações sobre doenças autorreferidas e hábitos de vida, tais como uso de bebidas alcoólicas, tabagismo, atividades de lazer e práticas de atividades físicas, além do Self-Reporting Questionnaire (SRQ-20), instrumento de triagem de morbidade psíquica utilizado para o rastreamento dos TMC, desenvolvido pela Organização Mundial da Saúde (OMS) ${ }^{4}$. O banco de dados foi construído e analisado utilizando-se o programa estatístico Statistical Package for the Social Sciences (SPSS) for Windows, versão 9.0.

Inicialmente, foi realizada a caracterização da população em estudo, quando foram analisadas as características sociodemográficas, TMC e as atividades físicas de lazer. Em seguida, estimou-se a prevalência de TMC global e de acordo com a realização de atividades físicas de lazer. Depois, foram realizadas associações entre TMC e as variáveis de interesse. Para tanto, foi utilizada como medida de associação a razão de prevalência (RP) e seus respectivos intervalos de confiança. Como medida de significância estatística, utilizou-se o teste de qui-quadrado de Pearson $\left(\chi^{2}\right)$, adotando-se $\mathrm{p} \leq 0,05$ para avaliação do nível de significância estatística. O programa R versão 2.6 .2 foi utilizado nessa análise ${ }^{5}$.

O estudo foi submetido e aprovado pelo Comitê de Ética em Pesquisa (CEP) da Universidade Estadual de Feira de Santana (protocolo $\mathrm{n}^{\circ}$ 028/2008 - CAAE 0028.050.000-08). Os procedimentos adotados seguiram as recomendações da Lei $n^{\circ}$ 196/96, do Conselho Nacional de Saúde.

\section{Resultados}

Na população selecionada, 47,7\% eram jovens entre 18 e 24 anos, a maioria era do sexo feminino $(68,4 \%), 68,5 \%$ solteiros, $81,8 \%$ de raça/cor negra (autorreferidos como de 
cor parda ou preta) (Tabela 1). Com relação à escolaridade, $55,4 \%$ estavam concluindo ou ainda cursando o ensino médio, e apenas $6,5 \%$ cursavam o ensino superior.

Tabela 1 - Distribuição dos jovens (15-29 anos) segundo aspectos sociodemográficos (Feira de Santana, BA, 2007)

\begin{tabular}{lcc}
\hline & \multicolumn{2}{c}{ Frequências } \\
\cline { 2 - 3 } Variáveis & $\mathbf{n}$ & $\mathbf{\%}$ \\
\hline Idade & 324 & 23,1 \\
$15-17$ & 667 & 47,7 \\
$18-24$ & 409 & 29,2 \\
$25-29$ & & \\
Sexo & 958 & 68,4 \\
Feminino & 442 & 31,6 \\
Masculino & & \\
Situação conjugal* & 953 & 68,5 \\
Solteiro(a) & 414 & 29,8 \\
Casado(a)/união estável & 24 & 1,7 \\
Divorciado(a)/separado(a)/desquitado(a)/viúvo(a) & & \\
Escolaridade* & & 0,6 \\
$\quad$ Analfabeto/semianalfabeto & 08 & 37,5 \\
Ensino fundamental & 524 & 55,4 \\
Ensino médio & 774 & 6,5 \\
Ensino superior & 91 & \\
Cor da pele* & & \\
Parda/preta & 1.096 & 81,8 \\
Branca & 226 & 16,9 \\
Amarela (oriental) & 12 & 0,9 \\
Origem indígena & 05 & 0,4 \\
\hline
\end{tabular}

* Informações perdidas: 9 para situação conjugal, 3 para escolaridade e 61 para cor da pele.

A participação em atividades de lazer entre os jovens de Feira de Santana foi elevada: 89,9\% afirmaram participar de alguma atividade regular de lazer para se distrair ou relaxar (Tabela 2). Quando questionados sobre o tempo de lazer disponível, 76,7\% dos jovens afirmaram que seu tempo para essas atividades era suficiente.

Tabela 2 - Distribuição de jovens (15-29 anos) segundo a prática de atividades de lazer (Feira de Santana, BA, 2007)

\begin{tabular}{lcr}
\hline & \multicolumn{2}{c}{ Frequências } \\
\cline { 2 - 3 } Atividades de lazer & $\mathbf{n}$ & $\mathbf{\%}$ \\
\hline Participa em atividades regulares de lazer & & \\
$\quad$ Sim & 1.259 & 89,9 \\
$\quad$ Não & 141 & 10,1 \\
Tempo para o lazer* & & \\
$\quad$ Suficiente & 939 & 76,7 \\
$\quad$ Insuficiente & 286 & 23,3 \\
Esforço físico despendido & & \\
$\quad$ Leve & 795 & 63,3 \\
Moderada & 329 & 26,2 \\
Pesada & 132 & 10,5 \\
\hline
\end{tabular}

* Informações perdidas: 34
As atividades de lazer foram classificadas, quanto ao esforço físico utilizado para praticá-las, em leves (lê, ouve rádio, assiste TV), moderadas (caminha, anda de bicicleta, faz aula de dança ou outra atividade física por pelo menos 2 horas semanais) ou pesadas (corre, faz ginástica, natação, jogos com bola ou atividade física, por pelo menos 4 horas semanais). Entre os jovens investigados, 63,3\% classificaram suas atividades de lazer como leves (Tabela 2).

A análise da intensidade da atividade de lazer praticada de acordo com a faixa etária mostrou que apenas 7,7\% dos jovens-jovens (18-24 anos) e 9,4\% dos jovens-adultos (25-29 anos) participavam de atividades de lazer consideradas pesadas quanto ao esforço físico utilizado para praticá-las, percentual bem menor quando comparado com os $17,7 \%$ observados entre adolescentes-jovens (15-17 anos). Portanto, o perfil de prática de atividades de lazer observado teve como característica o baixo gasto energético, que sugere uma alta tendência à inatividade física nos jovens feirenses, e que vai se agravando com o avançar da idade.

As atividades físicas de lazer praticadas pela amostra estão listadas na Tabela 3. Os adolescentes-jovens e os jovens-jovens participavam mais de atividades de lazer do que os jovens-adultos, com frequências de 91,0\% (295/324), 91,3\% (609/667) e 86,8\% (355/409), respectivamente. Esses achados podem ser justificados pelo acúmulo de atividades que os jovens vão assumindo com o passar do tempo, alternando o seu dia a dia entre trabalho e estudo, pouco tempo reservado para a prática de atividades físicas de lazer.

Tabela 3 - Distribuição de jovens (15-29 anos) de acordo com as atividades físicas de lazer (Feira de Santana, BA, 2007)

\begin{tabular}{lcc}
\hline & \multicolumn{2}{c}{ Frequências } \\
\cline { 2 - 3 } Atividades físicas & $\mathbf{n}$ & $\mathbf{\%}$ \\
\hline Ginástica & & \\
Sim & 84 & 6,6 \\
Não & 1.175 & 93,4 \\
Caminhada & & \\
$\quad$ Sim & 154 & 12,2 \\
Não & 1.105 & 87,8 \\
Futebol/vôlei & & \\
Sim & 292 & 23,2 \\
Não & 967 & 76,8 \\
Natação/bicicleta & & \\
Sim & 62 & 4,9 \\
Não & 1.197 & 95,1 \\
Hidroginástica & & \\
Sim & 05 & 0,3 \\
Não & 1.254 & 99,7 \\
Ioga/dança & & \\
Sim & 34 & 2,6 \\
Não & 1.225 & 97,4 \\
Outras* & & \\
Sim & 42 & 3,4 \\
Não & 1.217 & 96,6 \\
\hline
\end{tabular}

* Musculação, alongamento, basquete, boxe, capoeira, educação física, futsal, handebol, caratê, skate, tênis, etc. 
Atividade física de lazer e transtornos mentais comuns - Rios ET AL.

Tabela 4 - Transtornos mentais comuns segundo faixa etária (Feira de Santana, BA, 2007)

\begin{tabular}{|c|c|c|c|c|c|c|c|c|}
\hline \multirow[b]{2}{*}{ TMC* } & \multicolumn{2}{|c|}{$15-17$} & \multicolumn{2}{|c|}{$18-24$} & \multicolumn{2}{|c|}{ 25-29 } & \multicolumn{2}{|c|}{ Total } \\
\hline & $\mathrm{n}$ & $\%$ & $\mathrm{n}$ & $\%$ & $\mathrm{n}$ & $\%$ & n & $\%$ \\
\hline Não & 256 & 80,8 & 484 & 73,4 & 289 & 72,3 & 1.029 & 74,7 \\
\hline Sim & 61 & 19,2 & 175 & 26,6 & 111 & 27,8 & 347 & 25,3 \\
\hline Total & 317 & 100,0 & 659 & 100,0 & 400 & 100,0 & 1.376 & 100,0 \\
\hline
\end{tabular}

$\mathrm{TMC}=$ transtornos mentais comuns.

* Informações perdidas: 24 indivíduos $(1,7 \%)$.

Dentre as atividades socioculturais, assistir TV foi a atividade mais praticada pelos jovens $(97,1 \%)$, seguida de ouvir rádio $(88 \%)$ e visitar amigos $(83,6 \%)$. As altas frequências de assistir TV e ouvir rádio demonstraram o alto poder da mídia sobre a atenção dos jovens, além da predileção destes por atividades sedentárias.

Avaliando a prevalência de transtornos mentais, detectou-se uma relação entre TMC e faixa etária (Tabela 4). O percentual mais alto de TMC foi observado na faixa de 25 a 29 anos $(27,8 \%)$, e o menor percentual, na faixa de 15 a 17 anos $(19,2 \%)$.

Observou-se elevada prevalência de TMC (39,9\%) entre os jovens que afirmaram não participar de atividades regulares de lazer para distração ou relaxamento. A diferença observada mostrou-se estatisticamente significativa ( $\mathrm{RP}=$ 0,59, intervalo de confiança de 95\%: 0,51-0,67). Os indivíduos que participavam de atividades regulares de lazer apresentaram menor prevalência de TMC, revelando, assim, que a realização de tais atividades pode ser fator relevante para a promoção da saúde mental dos jovens.

\section{Discussão}

De acordo com os resultados apresentados, constatou-se que as atividades físicas e de lazer são elementos importantes frente às morbidades psíquicas. Entre os entrevistados de faixas etárias mais jovens, a participação em atividades de lazer foi mais frequente, e percebeu-se que o hábito diminuiu com o avançar da idade.

Com relação à intensidade das atividades de lazer, predominou a participação em atividades consideradas leves, que exigiam o mínimo de esforço físico, demonstrando que os jovens feirenses são, em sua maioria, sedentários no lazer.

O sedentarismo, reconhecidamente um fator de risco para doenças crônicas não transmissíveis, apresenta prevalência elevada em vários países ${ }^{6}$. Estudos indicam que grande parcela da população não atinge as recomendações atuais quanto à prática de atividades físicas. Além disso, ao avaliar apenas as atividades físicas realizadas no tempo de lazer, pesquisadores têm detectado prevalências de sedentarismo ainda mais elevadas ${ }^{7-9}$.

Duas categorias podem influenciar os padrões de atividade física: as características individuais, incluindo motivações, autoeficácia, habilidades motoras e outros comportamentos de saúde; e as características ambientais, como acesso ao trabalho ou a espaços de lazer, custos, barreiras de disponibilidade temporal e suporte sociocultural ${ }^{10}$.

Quanto às atividades socioculturais de lazer, a opção de visita a amigos é uma opção muito frequente nessa faixa etária, fato verificado também no estudo de Bonfim et al. ${ }^{11}$, que observaram que as articulações entre os jovens constituem uma busca pelo processo de construção de suas identidades juvenis através da sociabilidade. A troca de experiências e o compartilhamento dos medos e anseios vivenciados nessa fase da vida proporcionam a sociabilidade e a consequente identificação entre esses jovens.

A prevalência global de TMC entre os jovens neste estudo foi elevada, constituindo uma preocupação relevante para a saúde pública, tendo em vista que os TMC afetam negativamente a saúde das pessoas. Nesse sentido, ações em saúde mental direcionadas para a população de adolescentes e adultos são imprescindíveis.

Além disso, foi considerada alta a prevalência de TMC entre os jovens que afirmaram não participar de atividades de lazer (39,9\%). Ao analisar a associação entre participação em atividades de lazer e TMC, foi encontrada uma associação negativa estatisticamente significante.

Os resultados do presente estudo corroboram os achados da literatura de que adolescentes e jovens que não participam ou que participam insuficientemente de atividades físicas de lazer apresentam índices elevados de transtornos psiquiátri$\cos$ menores e/ou outros sofrimentos psíquicos ${ }^{12,13}$. Zaitune et al. ${ }^{14}$ também verificaram associação positiva entre TMC e sedentarismo no lazer, apontando que o sedentarismo contribui para o risco de depressão, de ansiedade e de prejuízos para a saúde mental. Peluso \& Andrade $^{15}$, por sua vez, afirmam que inúmeros benefícios psicossociais resultam da prática regular de atividade física de lazer, como a melhora do humor e da autoestima e a redução da ansiedade e da depressão.

Aliadas ao agitado cotidiano da sociedade moderna, as fases de transição (de crescimento e amadurecimento) pelas quais passam os jovens já são, em si, causas de sofrimento psíquico. Medos, ansiedades, angústias e inseguranças misturam-se aos sonhos e desejos de uma vida de sucesso, feliz e prazerosa, a qual, muitas vezes, é inviabilizada pelas desigualdades presentes na sociedade em que vivem.

$\mathrm{O}$ indivíduo, ao participar de atividades de lazer, sejam elas físicas ou socioculturais, acaba por fazer parte de um círculo social e promover a sua sociabilidade, além de se beneficiar de uma melhor saúde, tanto mental quanto física. $\mathrm{O}$ lazer e as atividades físicas funcionariam, portanto, como 
descarregadores de estresse, ansiedade e depressão, dentre outros TMC, promovendo a melhoria do bem-estar e da autoestima do cidadão e evitando seu isolamento social.

Entre as limitações deste estudo, pode-se apontar o próprio delineamento de pesquisa: o fato de este ser um estudo transversal nos impede de assegurar relações implícitas de causalidade entre as variáveis estudadas. No entanto, com base nos dados encontrados na literatura sobre o grau de associação e relações entre as variáveis estudadas, é possível dizer que os resultados aqui encontrados parecem suportar as evidências dos modelos explicativos de geração dos TMC na população.

\section{Conclusão}

Este estudo focaliza a prevalência de TMC e a importância da prática de atividades físicas de lazer para a saúde dos jovens, evidenciando a relevância do tema no contexto da saúde mental, ao se considerar as atividades físicas de lazer como fatores importantes na prevenção e no tratamento dos TMC. A participação em atividades físicas de lazer apresentou relação inversa com a prevalência de TMC e diminuiu consideravelmente com o avançar da idade. Nesse sentido, iniciativas de incentivo à participação em atividades físicas de lazer, assim como a construção e manutenção de equipamentos de lazer adequados (quadras, parques, cinemas), parecem ser alternativas viáveis e podem representar um ganho significativo à qualidade de vida da população e ao efeito benéfico que as atividades físicas e de lazer podem desempenhar nesse processo.

\section{Agradecimentos}

Ao Ministério da Saúde (convênio 1532/2005) e à Fundação de Amparo à Pesquisa do Estado da Bahia (FAPESB; processo $n^{\circ} 71 / 2004$ ) pelos recursos financeiros recebidos para a realização deste estudo.

\section{Referências}

1. Goldberg D Huxley P. Common mental disorders: a bio-social model. London/ New York: Tavistock/Routledge; 1992.

2. Brasil, Conselho Nacional de Juventude. Política Nacional de Juventude: diretrizes e perspectivas. São Paulo: Fundação Friedrich Ebert; 2006.

3. Instituto Brasileiro de Geografia e Estatística. Síntese de indicadores sociais, 2004. Rio de Janeiro: IBGE; 2006. Disponível em: http://www.ibge.gov.br/ home/estatistica/populacao/condicaodevida/indicadoresminimos/sinteseindicsociais2004/indic_sociais2004.pdf. Acessado 18 ago 2008.

4. World Health Organization. User's guide to the Self-Reporting Questionnaire (SRQ). Geneva: WHO; 1994.

5. The R Project for Statistical Computing. R: A language and environment for statistical computing. Vienna: R Foundation for Statistical Computing; 2008. http:// www.r-project.org.

6. Martinez-Gonzalez MA, Varo JJ, Santos JL, De Irala J, Gibney M, Kearney J, et al. Prevalence of physical activity during leisure time in the European Union. Med Sci Sports Exerc. 2001;33(7):1142-6.

7. Monteiro CA, Conde WL, Matsudo SM, Matsudo VR, Bonsenor IM, Lotufo PA. A descriptive epidemiology of leisure-time physical activity in Brazil, 19961997. Rev Panam Salud Publica. 2003;14(4):246-54.

8. Barros MV, Nahas MV. Comportamentos de risco, auto-avaliação do nível de saúde e percepção de estresse entre trabalhadores da indústria. Rev Saude Publica. 2001;35(6):554-63.

9. Dias-da-Costa JS, Hallal PC, Wells JC, Daltoe T, Fuchs SC, Menezes AM, et al. Epidemiology of leisure-time physical activity: a population-based study in Southern Brazil. Cad Saude Publica. 2005;21(1):275-82.

10. Camões $\mathrm{M}$, Lopes $\mathrm{C}$. Fatores associados à atividade física na população portuguesa. Rev Saude Publica. 2008;42(2):208-16.

11. Bonfim MCA, Sousa VMPB, Vieira EC. Juventudes e identidades: práticas culturais de jovens urbanos de Teresina na construção de suas identidades. Teresina: Universidade Federal do Piauí; 2006.

12. Oehlschlaeger MHK, Pinheiro RT, Horta B, Gelatti C, San'Tana P. Prevalência e fatores associados ao sedentarismo em adolescentes de área urbana. Rev Saude Publica. 2004;38(2):157-63.

13. Asztalos M, Wijndaele K, De Bourdeaudhuij I, Philippaerts R, Matton L, Duvigneaud $\mathrm{N}$, et al. Specific associations between types of physical activity and components of mental health. J Sci Med Sport. 2009;12(4):468-74.

14. Zaitune MPA, Barros MBA, César CLG, Carandina L, Goldbaum M. Fatores associados ao sedentarismo no lazer em idosos, Campinas, São Paulo, Brasil. Cad Saude Publica. 2007;23(6):1329-38.

15 Peluso MAM, Andrade LHSG. Physical activity and mental health: the association between exercise and mood. Clinics. 2005:60:61-70. 